\title{
Selective recognition of ATP by multivalent nano-assemblies of bisimidazolium amphiphiles through "turn-on" fluorescence response
}

\author{
Rakesh Biswas, Surya Ghosh, Shubhra Kanti Bhaumik and Supratim Banerjee*
}

\author{
Full Research Paper \\ Address: \\ Department of Chemical Sciences, Indian Institute of Science \\ Education and Research Kolkata, Mohanpur-741246, Nadia, India \\ Email: \\ Supratim Banerjee* - supratim.banerjee@iiserkol.ac.in \\ * Corresponding author \\ Keywords: \\ amphiphile; ATP; excimer; multivalency; self-assembly
}

\author{
Beilstein J. Org. Chem. 2020, 16, 2728-2738. \\ https://doi.org/10.3762/bjoc.16.223 \\ Received: 01 August 2020 \\ Accepted: 23 October 2020 \\ Published: 10 November 2020 \\ This article is part of the thematic issue "Molecular recognition" and is \\ dedicated to the memory of Carsten Schmuck. \\ Guest Editor: J. Niemeyer \\ (C) 2020 Biswas et al.; licensee Beilstein-Institut.
}

License and terms: see end of document.

\begin{abstract}
Bisimidazolium receptors, tagged with chromophoric pyrene at one end and linked to an $n$-alkyl chain at the other, underwent selfassembly in aqueous media depending on the length of the alkyl segment. The amphiphilic derivatives having $n$-decyl or longer chains, formed nano-assemblies with cyanic-green emission resulting from the stacked pyrene chromophores in the aggregates. The presence of positive surface charges on the multivalent aggregates led to ATP binding which was accompanied by a significant increase in the excimeric emission intensity. This provided a convenient way of monitoring ATP binding in a "turn-on" mode and an efficient detection of ATP was achieved in aqueous buffer and also in buffer containing $150 \mathrm{mM} \mathrm{NaCl}$ at physiological $\mathrm{pH}$ value. Furthermore, the multivalent aggregates demonstrated a significant selectivity in ATP detection over ADP, AMP and pyrophosphate.
\end{abstract}

\section{Introduction}

Supramolecular anion sensing is an active area of research which has developed tremendously in the last few decades [1-4]. The importance of anion sensing stems from its importance in various practical applications which range from identifying and detecting environmentally toxic anions to medical diagnostics. In recent years, anion responsive materials $[5,6]$ have also emerged as an interesting class of stimuli-responsive materials. Among the different varieties of anionic species, the selective and sensitive detection of bio-anions is particularly important due to their involvement in various biological functions. One of the challenges in the bio-anion detection is that the sensory probes have to be effective under physiological conditions. The highly polar nature of the aqueous media considerably weakens supramolecular interactions such as H-bonding 
and electrostatic interactions and as a result, it is in general challenging to design high-affinity synthetic probes for anion sensing under such competitive conditions [7]. One of the strategies that has proven to be quite successful in this regard, not only for bio-anions, rather for a variety of several other analytes also, is multivalency [8-11]. It is a principle ubiquitously used in biology to achieve high affinity binding events with examples ranging from protein-carbohydrate interactions to host-pathogen interactions or cell surface adhesion [12-14]. The high affinity originates from the simultaneous interactions of multiple sites in the receptor with their complementary binding partners in the target analyte [15]. One of the ways to design multivalent systems is to connect the receptors through covalent linkages. Conjugated polymers and conjugated polymer electrolytes are prominent examples of covalently constructed multivalent systems and they have been frequently exploited for the highly sensitive detection of a diverse range of target analytes [16-18]. However, the covalent route often requires time consuming synthetic steps and hence, in recent years, self-assembled multivalency has emerged as a suitable alternative [19]. In this methodology, multivalent arrays are built from comparatively smaller binding sites through selfassembly. The smaller molecular units are easier to synthesize and moreover, the morphology and the surface functionalities of the resultant multivalent structures can be tuned in a modular fashion [20,21]. A number of elegant examples of self-assembled multivalent systems targeting biological analytes such as DNA, heparin, proteins, carbohydrates, etc. have been reported in the literature [22-27].

ATP is an important bio-anion that is the energy currency in cells and is involved in a number of essential biological functions including active transport and cell division [28,29]. For ATP detection, metal complexes using metal ions such as $\mathrm{Zn}(\mathrm{II}), \mathrm{Cu}(\mathrm{II})$ and lanthanide ions have been frequently employed [30-35]. A variety of other systems such as aptamers
[36-38], conjugated polymers [39-42], quantum dots [43,44] and sensors based on organic receptors such as imidazolium, ammonium, guanidinium [45-52], etc. have also been developed. Imidazolium based synthetic receptors typically utilize a combination of electrostatic and $(\mathrm{C}-\mathrm{H}) \cdots \mathrm{O}^{-}$hydrogen bonds involving the acidic proton at the 2-position of the imidazolium moiety to bind to ATP and other phosphate analytes [46]. A number of cyclophanes and tweezers [53-56] have been reported possessing the following common structural features: a) the presence of multiple imidazolium groups to overcome the high hydration enthalpy of phosphates and b) the imidazolium groups are connected to aromatic moieties such as anthracene, pyrene, etc. The variation in the luminescence of the aromatic moieties signals the binding event and furthermore, they provide additional stacking interactions with the nucleobases which contributes to the stability of the complexes. In many cases, these stacking interactions provide binding selectivity among the nucleotides [53-56].

Most of the aforementioned receptors were predominantly "molecular" in nature with the binding of the nucleotides occurring through multivalent electrostatic interactions in the cavity/cleft created by the spatial orientation of the imidazolium groups. We herein consider a self-assembly based approach in which amphiphilic monomers with bisimidazolium moieties are utilized to create multivalent nano-aggregates for binding to the nucleotides. For this purpose, four pyrene tagged bisimidazolium-based receptors PBImN (Figure 1, $N=4,10,12$ and 14; $N$ denotes the number of carbons in the $n$-alkyl chain) were synthesized. The longer chain derivatives with $N \geq 10$ underwent self-assembly in aqueous buffer and produced nanoaggregates with surface exposed positive charges. Interestingly, the aggregates displayed cyanic green luminescence which originated from the excimeric emission of the stacked pyrenes [57-59]. In a recent work, we have demonstrated that self-assembled nanofibrous aggregates of anthracene tagged imidazolium re-

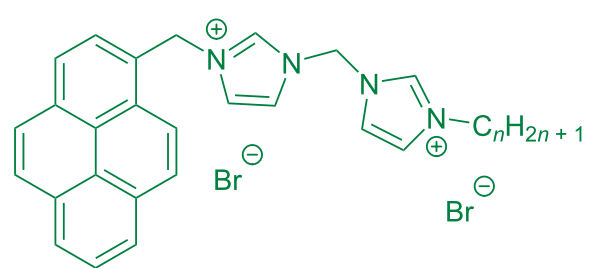

PBImN<smiles>CC(C)OP(=O)(O)OCC1OC(n2cnc3c(N)ncnc32)C(O)C1O</smiles>

$n=1 ; \quad$ AMP

$n=2 ; \quad$ ADP

$n=3 ; \quad$ ATP 
ceptors responded to ATP through an amplified fluorescence quenching [60]. Based on these results, we wanted to develop similar self-assembled systems which would show a "turn-on" response in the presence of ATP or other phosphate analytes. The current design of PBImNs is based on this objective and we found that the excimeric pyrene emissions in these aggregates indeed exhibited a significant increase in the presence of ATP and thus provided a convenient protocol for its detection (Scheme 1). Furthermore, this "turn-on" response was achieved with an appreciable level of selectivity as the response observed in the presence of other adenosine phosphates, ADP and AMP and even pyrophosphate (PPi) was comparatively much lower than that observed for ATP in a wide concentration range $(1-100 \mu \mathrm{M})$.

\section{Results and Discussion Synthesis}

The synthesis of $\operatorname{PBImNs}(N=4,10,12,14)$ is depicted in Scheme 2 and the elaborate procedures are given in Supporting Information File 1. The preparation of compounds $\mathbf{2}$ and $\mathbf{3}$ are analogous to the procedures from ref. [60]. The pyrene derivative 1-(bromomethyl)pyrene (3), which was the common starting material for making all the PBImNs, was synthesized from pyrene-1-carbaldehyde (1) following a literature report [46]. In brief, pyrene-1-carbaldehyde (1) was first converted to pyren-1-ylmethanol (2) by reduction with sodium borohydride in ethanol [61] and this was followed by treating of 2 with $\mathrm{PBr}_{3}$ in toluene to furnish 3. Di(1H-imidazol-1-yl)methane (5) was synthesized from imidazole (4) and $\mathrm{CH}_{2} \mathrm{Br}_{2}$ following a litera- ture procedure $[62,63]$ with slight modifications. Then, BImNs $(N=4,10,12,14)$ were synthesized from 5 by mono-alkylation using the corresponding $n$-alkyl halides in DMF. Finally, PBImNs $(N=4,10,12,14)$ were obtained through nucleophilic substitution reactions between $\operatorname{BIm} N \mathrm{~s}(N=4,10,12,14)$ and 3. All synthesized compounds were characterized by NMR spectroscopy and high-resolution mass spectrometry.

\section{Self-assembly properties of PBImNs in aqueous buffer}

The self-assembly properties of the four $\operatorname{PBIm} \boldsymbol{N}$ derivatives in aqueous buffer ( $5 \mathrm{mM}$ tris- $\mathrm{HCl}, 10 \mathrm{mM} \mathrm{NaCl}$, water/DMSO 99.5:0.5, $\mathrm{pH}$ 7.4) were investigated using a combination of absorption and emission spectral studies, dynamic light scattering (DLS) and field emission scanning electron microscopy (FESEM). In a "good solvent" such as DMSO, in which all PBImNs were readily soluble, almost identical absorption $\left(\lambda_{\max }=347 \mathrm{~nm}\right)$ and emission $\left(\lambda_{\max }=395 \mathrm{~nm}\right)$ spectra were observed (Supporting Information File 1, Figure S1). In comparison, in aqueous buffer, certain differences existed between the butyl derivative PBIm4 and the three longer chain derivatives PBIm10, PBIm12 and PBIm14, hinting at the possible self-assembly behavior of the latter three molecules. In order to investigate in a detailed manner, we first carried out absorption measurements at different concentrations (Figure 2a, Supporting Information File 1, Figures S2a and S3a). All derivatives displayed three absorption peaks around 314-316 nm, 327-329 $\mathrm{nm}$ and 343-345 $\mathrm{nm}$. These peaks were assigned to $0-0$ and its vibronic replicas related to an $\mathrm{S}_{0}-\mathrm{S}_{2}$ transition in the

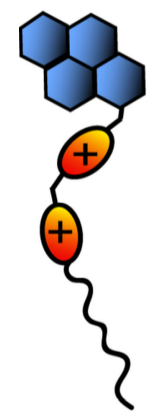

PBImN

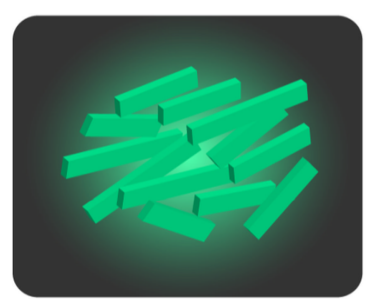

Self-assembled aggregates

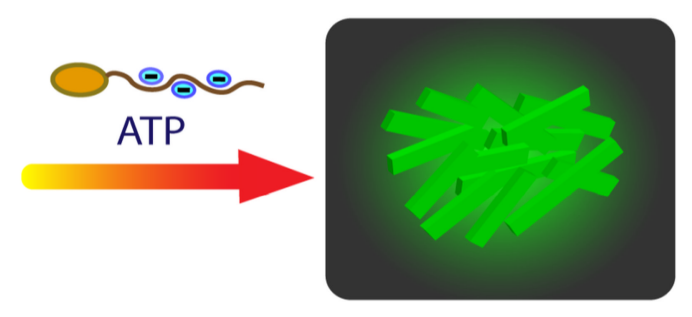

Multivalent binding
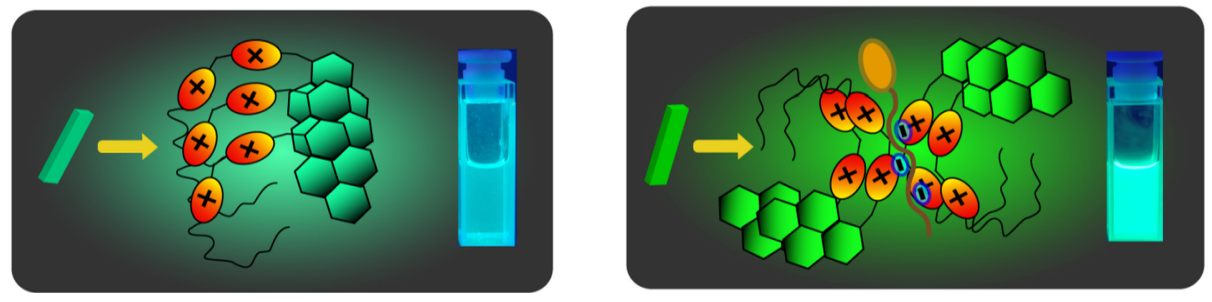

Scheme 1: Schematic representation of ATP sensing by multivalent assemblies of PBImN in aqueous media. 

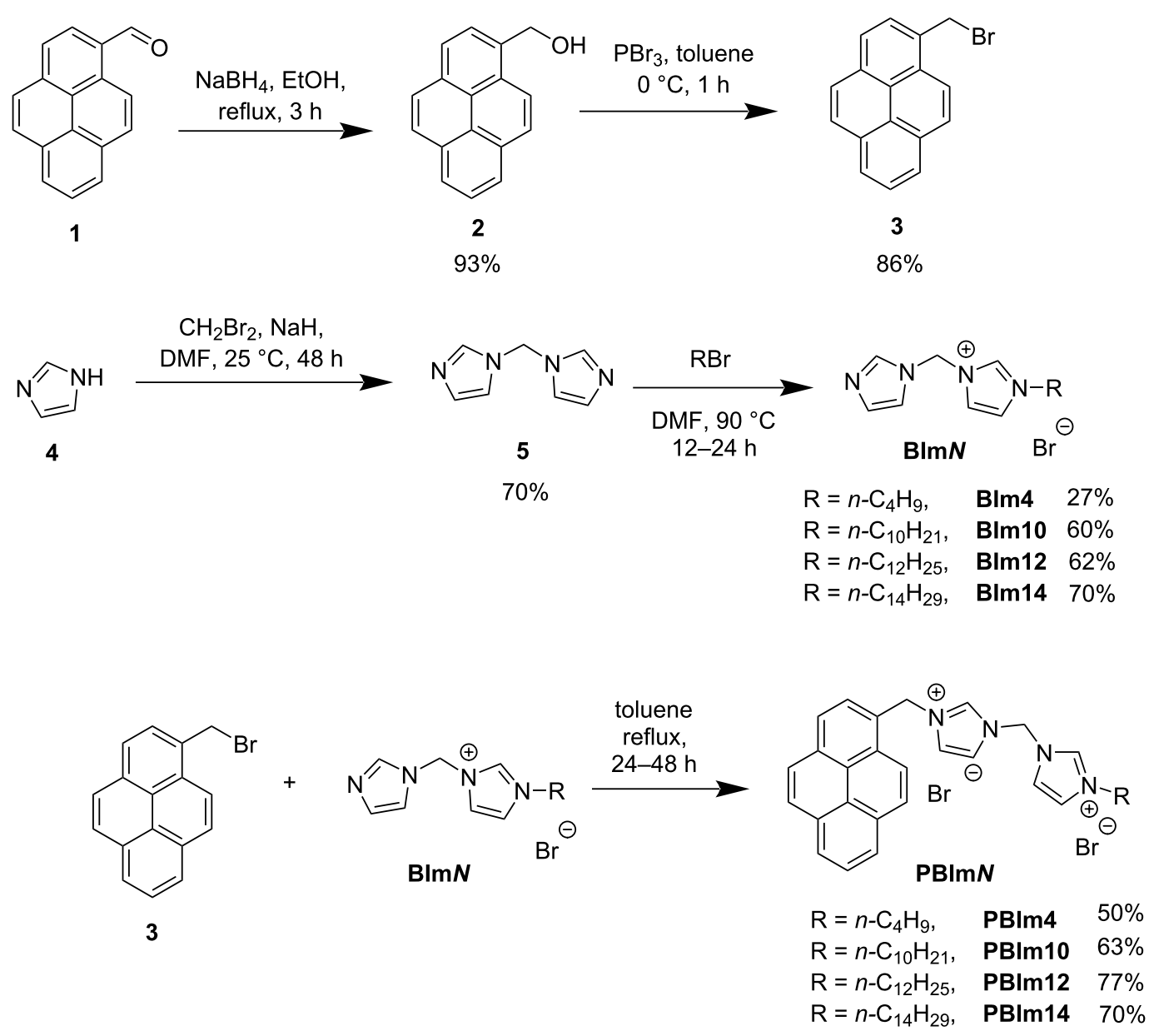

Scheme 2: Synthetic route for the preparation of PBImNs.

pyrene derivatives [64]. The concentration dependence of the $A_{0-0} / A_{0-1}$ ratio $\left(A_{0-0}\right.$ and $A_{0-1}$ are the absorbance of the $0-0$ and $0-1$ vibronic bands at $343-345 \mathrm{~nm}$ and $327-329 \mathrm{~nm}$, respectively) generally indicates a possible mode of organization of the pyrene chromophores upon self-assembly [63]. It was found that in the concentration range of 5-50 $\mu \mathrm{M}$ [65], although a decrease in this ratio was observed for PBIm4; much more pronounced reductions were noticed for the $N \geq 10$ derivatives (Figure 2a and Supporting Information File 1, Table S1), with the highest change being exhibited by PBIm14. These observations suggested that in going to higher concentrations, there was a gradual increase in the aggregation of the longer chain derivatives and in the aggregates, the pyrene chromophores had ground state interactions and were possibly stacked in co-facial manner as found in H-aggregates [63]. The aggregate formation was further supported by FESEM images (Figure 3a and Supporting Information File 1, Figure S7) which revealed the formation of elongated nano-objects for PBIm10, PBIm12 and PBIm14. The existence of the aggregates was also revealed by the DLS measurements. The three $N \geq 10$ deriva- tives exhibited nanometer size aggregates (Table 1) whereas PBIm4 did not exhibit any aggregate formation.

The emission spectra recorded in aqueous buffer (Figure 2b) also showed a significant difference between PBIm4 and the three amphiphilic derivatives. For example, the amphiphilic derivatives exhibited structured emission bands around $376 \mathrm{~nm}$, $396 \mathrm{~nm}$ and $417 \mathrm{~nm}$ as well as a broad structureless band centered around 480-490 nm (PBIm10: $481 \mathrm{~nm}$, PBIm12: $485 \mathrm{~nm}$ and PBIm14: $490 \mathrm{~nm}$ ). These were assigned to the characteristic monomeric and excimeric emissions of pyrene, respectively [58]. The intensity of the excimeric emission was found be higher with $365 \mathrm{~nm}$ excitation (Supporting Information File 1, Figure S5; see Figure S6 for the excitation spectra) and hence this wavelength was used for all the subsequent studies. In comparison, PBIm4 displayed a weaker broad band at $475 \mathrm{~nm}$ along with comparatively more intense monomeric bands. Since no aggregate was observed from the DLS studies in the studied concentration range, it meant that the excimeric emission presumably resulted from small clusters of PBIm4. 

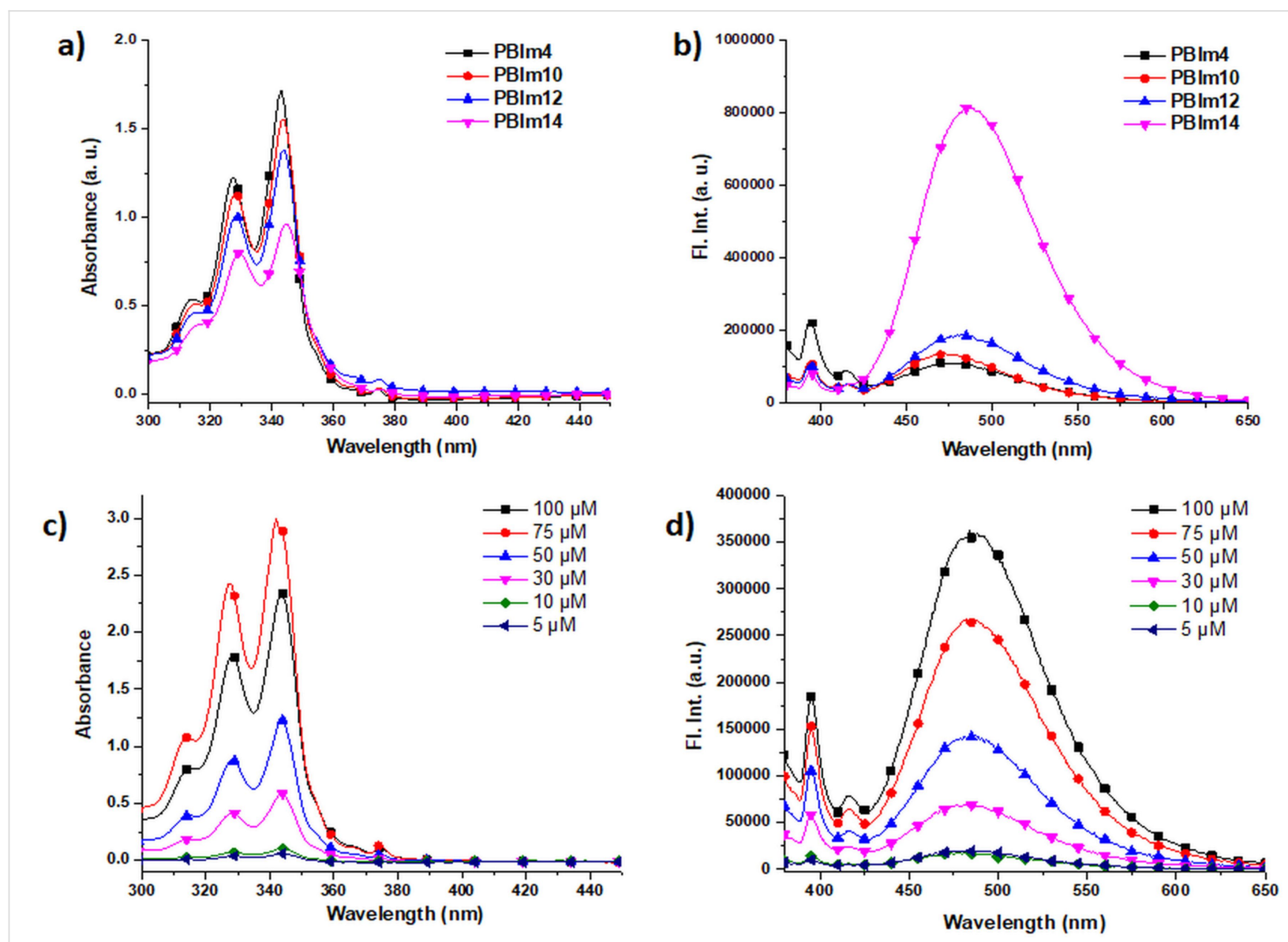

Figure 2: (a) Absorption and (b) emission spectra of PBImN (50 $\mu \mathrm{M})$ derivatives in buffer. (c) Absorption and (d) emission spectra of PBIm12 at different concentrations in buffer.

Time-correlated single photon counting (TCSPC) measurements revealed $\approx 80$ ns (Supporting Information File 1, Table S2) lifetimes for the $395 \mathrm{~nm}$ monomeric peak in DMSO for all the four derivatives and these values correlated well with the reported lifetimes for monomeric pyrenes [66,67]. In aqueous buffer, the lifetime of the $395 \mathrm{~nm}$ band decreased $(\approx 15-35 \mathrm{~ns}$, Supporting Information File 1, Table S3) probably owing to the increment in the solvent polarity [68]. The average lifetime values $\left(\tau_{\mathrm{avg}}\right.$ ) of the excimeric bands of PBIm10, PBIm12 and PBIm14 were found to be $33.97 \mathrm{~ns}$ (481 nm), $34.44 \mathrm{~ns}$ $(485 \mathrm{~nm})$ and $33.27 \mathrm{~ns}(490 \mathrm{~nm})$, respectively (Supporting Information File 1, Table S3). The large Stokes shifts and the absence of vibronic features are typical characteristics of excimeric emission of pyrene $[69,70]$.

\section{ATP binding studies}

The aggregates with $N \geq 10$ exhibited positive zeta potential ( $\zeta)$ values under the studied conditions (Table 1) suggesting that they possessed surface exposed positive charges. Based on this, we envisaged that they could be potential ATP binders in aqueous buffer. Indeed, upon addition of ATP, zeta potential $(\zeta)$ values were significantly reduced and eventually became negative (Table 1) indicating a strong interaction of the negatively charged ATP with the aggregates. An appreciable increase in the size of the nanoaggregates was also observed upon addition of ATP (Table 1) pointing to the formation of larger co-aggregates. Furthermore, a visible change in morphology for the co-aggregates was observed from FESEM images (Figure 3b). All these results, taken together, indicated that the nanoaggregates were able to recognize ATP in a multivalent fashion leading to the formation of larger co-aggregates. On the contrary, PBIm4 did not show the formation of aggregates even after the addition of ATP.

The most interesting feature of the ATP binding was the remarkable increase in the excimeric emission of the nanoaggregates (Figure 3c and Supporting Information File 1, Figure S8b and S8c). The sensitivity of the detection was correlated to the $I / I_{0}$ ratio ( $I$ and $I_{0}$ are the emission intensity of the excimeric bands with and without ATP). PBIm12 (75 $\mu \mathrm{M})$ displayed the best sensitivity among the three amphiphilic derivatives having a significantly enhanced $I / I_{0}$ value of 10.6 upon 


\section{a)}

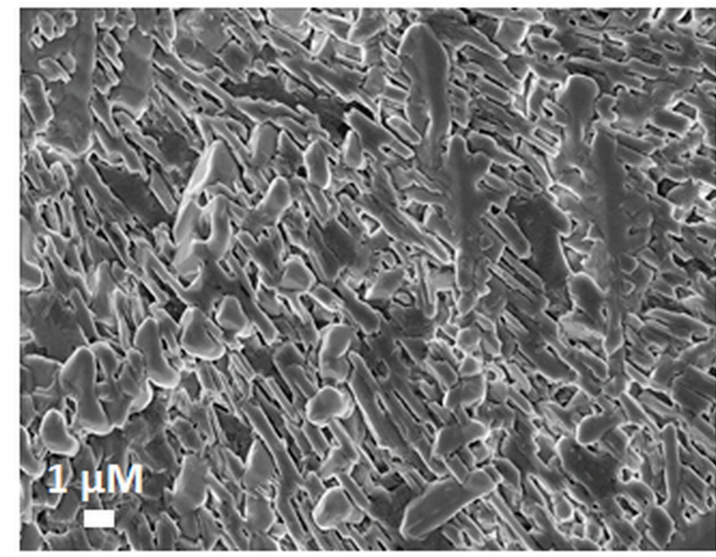

b)

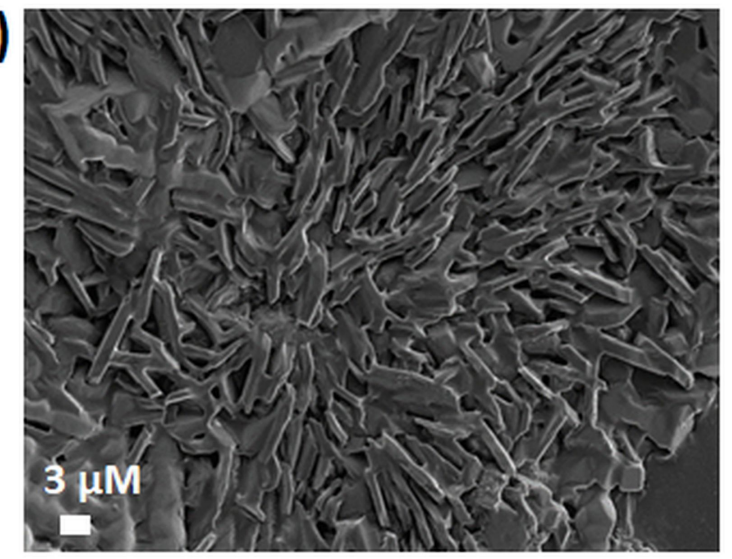

c)

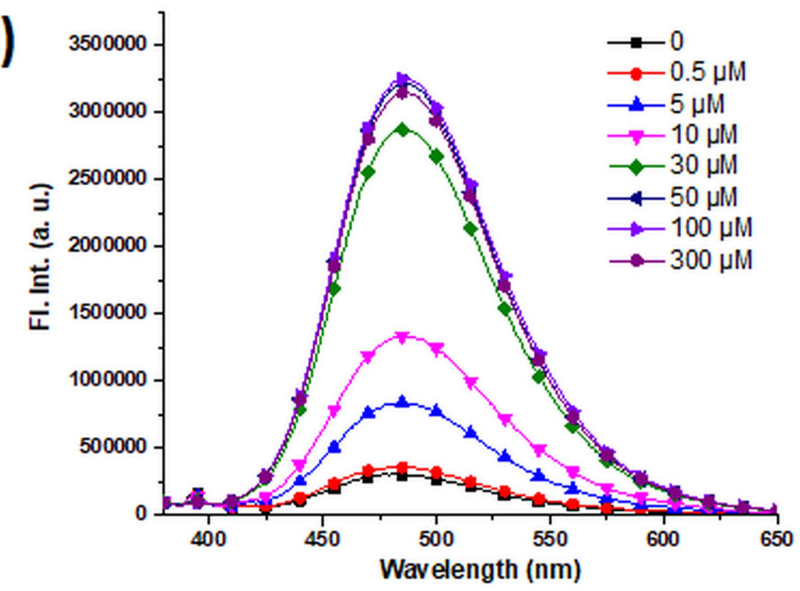

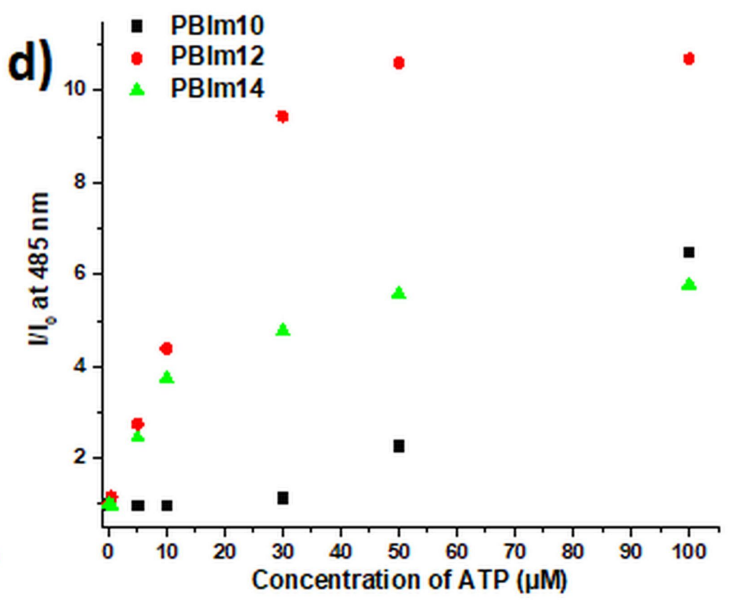

Figure 3: FESEM images of PBIm12 (a) without and (b) with ATP. (c) Emission spectral changes of PBIm12 (75 $\mu \mathrm{M})$ in buffer upon addition of ATP. (d) Comparison plot for $1 / /_{0}$ of PBIm10 $(100 \mu \mathrm{M})$, PBIm12 $(75 \mu \mathrm{M})$ and PBIm14 $(30 \mu \mathrm{M})$ upon addition of different amounts of ATP.

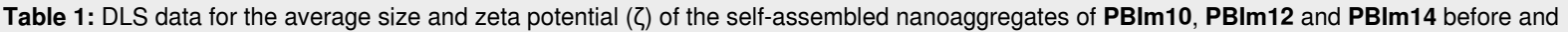
after ATP addition in aqueous buffer ( $5 \mathrm{mM}$ tris- $\mathrm{HCl}, 10 \mathrm{mM} \mathrm{NaCl}$, water/DMSO, 99.5:0.5, $\mathrm{pH}$ 7.4).

\begin{tabular}{ccccc} 
PBImN derivative & Size $(\mathrm{nm}) \mathrm{w} / \mathrm{o}-\mathrm{ATP}$ & $\begin{array}{c}\text { Size }(\mathrm{nm}) \mathrm{w} / \text { - ATP } \\
(50 \mu \mathrm{M})\end{array}$ & $\zeta(\mathrm{mV})$ w/o- ATP & $\begin{array}{c}\zeta(\mathrm{mV}) \mathrm{w} /-\mathrm{ATP} \\
(50 \mu \mathrm{M})\end{array}$ \\
\hline $\begin{array}{c}\text { PBIm10 } \\
(150 \mu \mathrm{M})\end{array}$ & 619.5 & 1582 & 3.45 & -10.1 \\
$\begin{array}{l}\text { PBIm12 } \\
(75 \mu \mathrm{M})\end{array}$ & 689.2 & 1449 & 2.41 & -8.02 \\
PBIm14 & & & 14 & -6.53
\end{tabular}

$50 \mu \mathrm{M}$ ATP addition whereas for PBIm10 $(100 \mu \mathrm{M})$ and PBIm14 $(30 \mu \mathrm{M})$ the values were 2.3 and 5.8 , respectively (Figure 3d). It is noteworthy to mention here that for each of the amphiphiles, the best response was obtained at an optimum concentration of the amphiphiles. For example, both at a concentration lower and higher than $75 \mu \mathrm{M}$, PBIm12 exhibited lower responses (Supporting Information File 1, Figure S9). In terms of the lower limit of detection, ATP was detected at $500 \mathrm{nM}$ concentration by the PBIm12 aggregates. A noticeable increase in the average lifetime $\left(\tau_{\mathrm{avg}}\right)$ of the excimeric band was observed after ATP addition (Supporting Information File 1, Table S4). For example, in case of PBIm12 (75 $\mu \mathrm{M})$, it increased from $34.44 \mathrm{~ns}$ to $41.99 \mathrm{~ns}$ upon ATP $(50 \mu \mathrm{M})$ addition (Supporting Information File 1, Table S4). ATP also induced sub- 
stantial changes in the absorption spectra for the amphiphilic derivatives (Supporting Information File 1, Figure S10b and 10c). The absorption maxima red shifted slightly and increased scattering was observed at higher amounts of ATP. The $A_{0-0} / A_{0-1}$ values were also found to decrease upon formation of the co-aggregates with ATP (Supporting Information File 1, Table S5).

The small-chain analogue, PBIm4 $(100 \mu \mathrm{M})$, on the contrary, showed only nominal changes in its monomeric or excimeric emission upon ATP addition (Supporting Information File 1, Figure S8a). It has already been pointed out that under these conditions, PBIm4 was mainly present in monomeric form along with some proportions of small clusters. Previous literature reports have shown that for "molecular" imidazoliumbased sensors, typically four positive charges $[46,54,56]$ were necessary to accomplish ATP detection in the micromolar concentration range. The lack of an ATP detection capability of PBIm4, therefore, clearly illustrated the point that under these conditions, ATP binding was difficult for a receptor with two positive charges either in "molecular" form or as clusters and further signified the requirement of a multivalent organization of the charges as provided by the aggregates.

\section{Selectivity in the ATP detection}

One of the key issues of a sensor design is the selectivity for an analyte in comparison to other interfering analytes. Therefore, we carried out selectivity studies with the multivalent sensors for ATP over the other adenosine phosphates ADP and AMP, PPi and inorganic phosphate (Pi). We employed PBIm12 $(75 \mu \mathrm{M})$ for this purpose as it exhibited the best sensitivity in the detection of ATP. A significant selectivity was noticed for ATP over ADP and AMP, PPi and Pi (Figure 4a and Figure 4b). For example, the $I / I_{0}$ value at $485 \mathrm{~nm}$ for PBIm12 $(75 \mu \mathrm{M})$ increased to 10.62 upon ATP addition $(50 \mu \mathrm{M})$, whereas for PPi $(50 \mu \mathrm{M})$ and $\operatorname{ADP}(50 \mu \mathrm{M})$, these values were 4.8 and 1.87 , respectively (Figure 4b). Practically no increments were observed for AMP $(50 \mu \mathrm{M})$ and $\mathrm{Pi}(50 \mu \mathrm{M})$ (Supporting Information File 1, Figure S11a and Figure 4b). As the multivalent binding is predominantly through electrostatic interactions, it
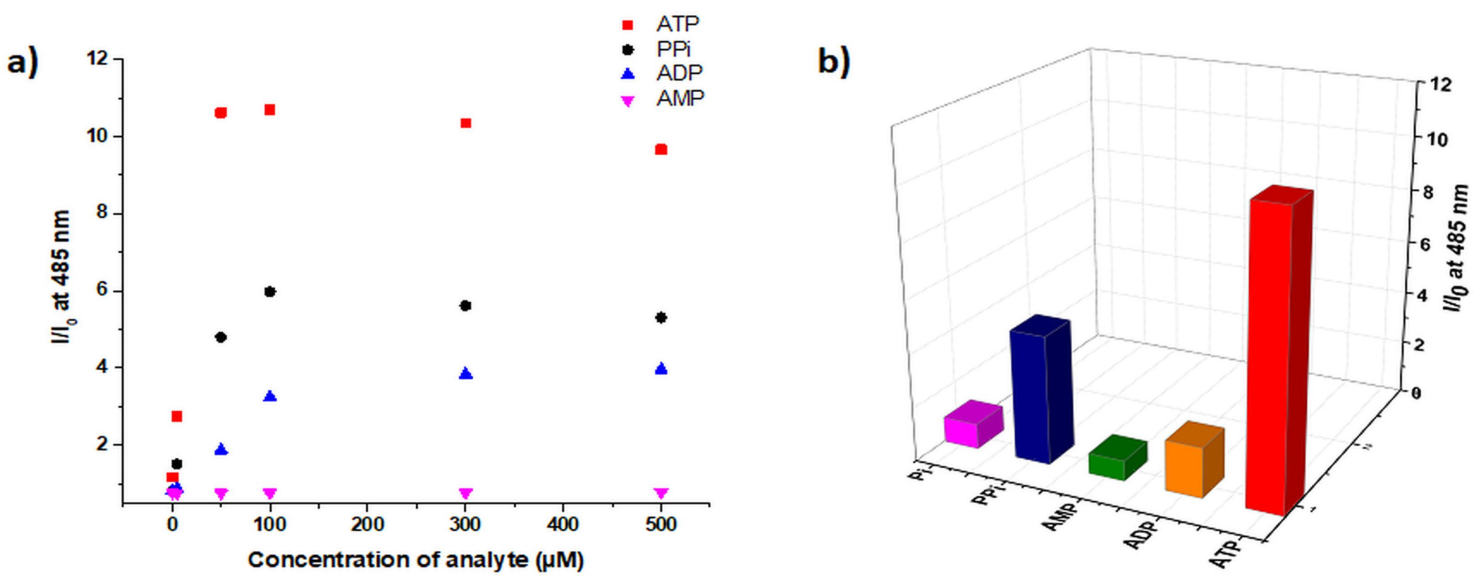

c)

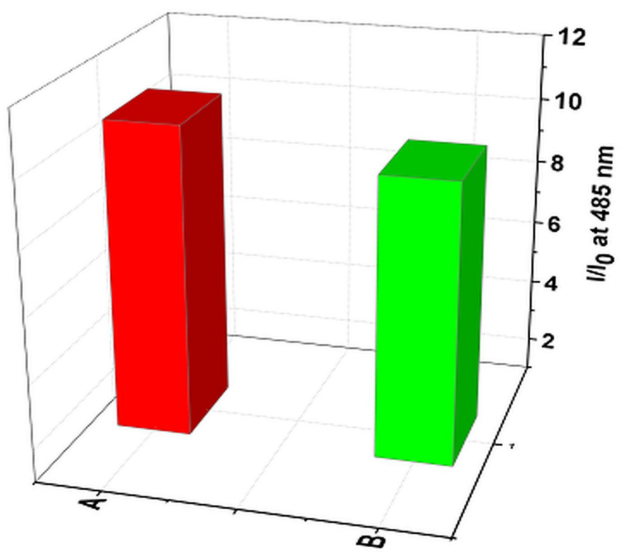

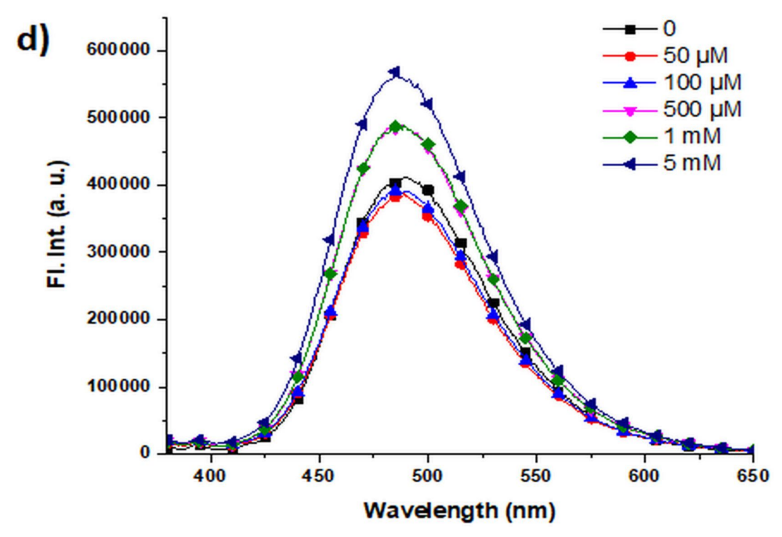

Figure 4: a) Emission changes of PBIm12 (75 $\mu \mathrm{M})$ upon the addition of ATP, ADP, AMP and PPi in buffer. Bar diagram comparing the $I / I_{0}$ values $(485 \mathrm{~nm})$ of PBIm12 $(75 \mu \mathrm{M})$; b) upon addition of ATP, ADP, AMP, PPi and Pi in a buffer (each at $50 \mu \mathrm{M})$ and c) with only ATP $(50 \mu \mathrm{M})(\mathbf{A})$ and a mixture of ATP, ADP, AMP, PPi and Pi (50 $\mu \mathrm{M}$ of each) (B) in buffer. d) Emission spectra of PBIm12 (100 $\mu \mathrm{M})$ upon the addition of ATP in a buffer containing $150 \mathrm{mM} \mathrm{NaCl}$. 
was not surprising to see a selectivity towards ATP (3 negative charges) over ADP and AMP (2 and 1 negative charge(s), respectively) at physiological $\mathrm{pH}$. However, it was intriguing to note the selectivity over PPi which had four negative charges. It is likely that in the ATP induced co-assemblies, there is a more compact arrangement of the stacked pyrene chromophores and this restriction of internal motion might have resulted in a higher excimer intensity [71]. In order to see whether the presence of other phosphate analytes interfered in ATP detection, fluorescence enhancements of PBIm12 $(75 \mu \mathrm{M})$, for only ATP $(50 \mu \mathrm{M})$ and ATP $(50 \mu \mathrm{M})$ in the presence of a mixture of ADP/AMP/PPi/Pi (50 $\mu \mathrm{M}$ of each analyte) were compared. As can be seen from Figure 4c, even in the presence of four other phosphate analytes with a total concentration of $200 \mu \mathrm{M}$, the $I / I_{0}$ ratio only showed a marginal decrease compared to that obtained in the presence of only ATP. This experiment thus clearly showed that the selectivity towards ATP was retained even in the presence of other phosphates. PBIm12 (75 $\mu \mathrm{M})$ was also able to discriminate ATP from GTP up to $30 \mu \mathrm{M}$ concentration (Supporting Information File 1, Figure S13b). The response of PBIm12 was also examined in the presence of many other anions such as iodide, bromide, fluoride, acetate, bicarbonate, sulfate, etc. and all of them exhibited only negligible changes in the excimeric emission (Supporting Information File 1, Figure S14).

\section{ATP detection in the presence of $150 \mathrm{mM}$ $\mathrm{NaCl}$}

In order to demonstrate the practical utility of the sensors, ATP binding studies were investigated in a highly competitive medium, i.e., a buffer containing $150 \mathrm{mM} \mathrm{NaCl}$. In a medium of such a high ionic strength, the binding affinity typically gets diminished by electrostatic screening [72]. Although the response was comparatively much lower than that observed in the plain buffer, ATP detection was possible in the concentration range of $200 \mu \mathrm{M}$ to $5 \mathrm{mM}$ using PBIm12 $(100 \mu \mathrm{M}$, Figure $4 \mathrm{~d})$ and this covered the typical concentration values of ATP in cell (3-5 mM) [73].

\section{Conclusion}

In conclusion, we present here the synthesis, self-assembly and phosphate binding properties of four imidazolium-based receptors $\operatorname{PBIm} N(N=4,10,12,14)$. The derivatives with $\mathrm{N} \geq 10$ chains were amphiphilic in nature and underwent self-assembly in aqueous buffer to produce nano-assemblies that displayed excimeric emission from the stacked pyrene chromophores. The positive surface potential values of the nano-assemblies allowed them to bind to ATP which was accompanied by a noticeable increase in the intensity of the excimeric emission. Furthermore, the multivalent assemblies displayed a high-level selectivity towards ATP over other adenosine phosphates (ADP and AMP) and pyrophosphate (PPi). The detection of ATP was also possible in the presence of $150 \mathrm{mM} \mathrm{NaCl}$. The modular nature of the sensing platform provided by the multivalent nanoassemblies of the bisimidazolium amphiphiles can in principle be extended for the detection of other biologically important analytes through a structural modulation of the amphiphiles and the studies along this direction are currently in progress in our group.

\section{Experimental Materials and methods}

All starting materials were purchased from commercially available sources and used without further purification. All the nucleotides, 1-pyrenecarboxaldehyde, sodium borohydride, sodium hydride, phosphorus tribromide and 1-bromobutane, 1-bromodecane, 1-bromododecane and 1-bromotetradecane were purchased from Sigma-Aldrich. Imidazole, dibromomethane and tris(hydroxymethyl)aminomethane were purchased from TCI chemicals. Sodium chloride, 28\% ammonia solution and all the solvents were purchased from Merck. Milli$\mathrm{Q}$ water was used for all the experiments. UV-vis spectroscopic measurements were carried out in Agilent Cary 8454 spectrophotometer. Emission spectroscopic measurements were carried out in a Horiba Fluoromax 4 spectrofluorometer. Images were taken under a $365 \mathrm{~nm}$ UV lamp. DLS and zeta potential measurements were carried out using a Malvern Zetasizer NanoZS. A Horiba Jobin Yvon Fluorocube instrument fitted with a $340 \mathrm{~nm}$ diode laser excitation source (with a temporal resolution of $70 \mathrm{ps}$ ) was used for the time-resolved fluorescence experiments applying the time correlated single photon counting (TCSPC) method. ${ }^{1} \mathrm{H}$ and ${ }^{13} \mathrm{C}$ NMR were performed on Jeol $400 \mathrm{MHz}$ and Bruker $500 \mathrm{MHz}$ spectrometers. Mass spectra were recorded in a Bruker mass spectrometer. SEM images was recorded by using the instrument CARL ZEISS (model SUPRA 55VP).

\section{Preparation of solutions}

\section{A. Preparation of PBImN solutions}

Initially, stock solutions of PBIm $N$ s $(N=4,10,12,14)$ were prepared by dissolving the solid powders in spectroscopic grade dimethyl sulfoxide (DMSO). These concentrated DMSO solutions were then diluted to $5 \mathrm{mM}$ tris- $\mathrm{HCl}$ buffer in $10 \mathrm{mM} \mathrm{NaCl}$ made in Milli-Q water to get the desired solutions having 1 or $0.5 \%$ DMSO in the final DMSO fraction. Solutions of PBIm4 in the buffer were equilibrated for $10 \mathrm{~min}$ whereas the solutions of PBIm10, PBIm12 and PBIm14 were equilibrated for $15 \mathrm{~min}$.

\section{B. Preparation of stock solutions of nucleotides}

Stock solutions of nucleotides $(10 \mathrm{mM})$ were prepared by dissolving the required amounts of them in Milli-Q water. 


\section{Titration procedures}

During the fluorometric titration, in every set of titrations a fresh solution of PBIm $N$ was prepared and equilibrated for $15 \mathrm{~min}$ and then the spectra were recorded.

\section{FESEM sample preparation}

Aqueous buffer solutions of PBImNs ( $N=10,12$ and 14) were first stabilized for $15 \mathrm{~min}$ and then $5 \mu \mathrm{L}$ of these solutions were drop casted on glass cover slips. The samples were dried in air overnight and then under high vacuum for $8-10$ hours. Finally, the morphologies of the samples were recorded in a FESEM instrument. Morphologies of ATP-added PBImNs samples

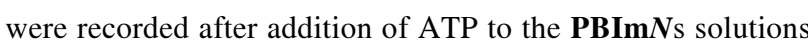
followed by drying the drop casted solutions of ATP-added PBImNs on glass cover slips in a similar way.

\section{Supporting Information}

\section{Supporting Information File 1}

Synthetic procedures, additional spectroscopic, microscopic and dynamic light scattering data (figures and tables) and NMR characterization.

[https://www.beilstein-journals.org/bjoc/content/ supplementary/1860-5397-16-223-S1.pdf]

\section{Funding}

IISER Kolkata and INSPIRE are acknowledged for research fellowships to RB and SKB, respectively. We are thankful to the Science and Engineering Research Board (SERB), Department of Science and Technology (DST), Government of India (project: EMR/2016/002838) and Indian Institute of Science Education and Research Kolkata for support of this work.

\section{ORCID ${ }^{\circledR}$ iDs}

Supratim Banerjee - https://orcid.org/0000-0001-8558-4444

\section{References}

1. Busschaert, N.; Caltagirone, C.; Van Rossom, W.; Gale, P. A. Chem. Rev. 2015, 115, 8038-8155. doi:10.1021/acs.chemrev.5b00099

2. Flood, A. H. Beilstein J. Org. Chem. 2016, 12, 611-627. doi:10.3762/bjoc. 12.60

3. Wu, J.; Zou, Y.; Li, C.; Sicking, W.; Piantanida, I.; Yi, T.; Schmuck, C. J. Am. Chem. Soc. 2012, 134, 1958-1961. doi:10.1021/ja2103845

4. Maity, D.; Schmuck, C. Chem. - Eur. J. 2016, 22, 13156-13161. doi:10.1002/chem.201602240

5. Maeda, H. Chem. - Eur. J. 2008, 14, 11274-11282. doi:10.1002/chem.200801333

6. Zhang, Z.; Kim, D. S.; Lin, C.-Y.; Zhang, H.; Lammer, A. D.; Lynch, V. M.; Popov, I.; Miljanić, O. Š.; Anslyn, E. V.; Sessler, J. L. J. Am. Chem. Soc. 2015, 137, 7769-7774. doi:10.1021/jacs.5b03131
7. You, L.; Zha, D.; Anslyn, E. V. Chem. Rev. 2015, 115, 7840-7892. doi:10.1021/cr5005524

8. Mammen, M.; Choi, S.-K.; Whitesides, G. M. Angew. Chem., Int. Ed. 1998, 37, 2754-2794. doi:10.1002/(sici)1521-3773(19981102)37:20<2754::aid-anie2754>3.0 co;2-3

9. Badjić, J. D.; Cantrill, S. J.; Stoddart, J. F. J. Am. Chem. Soc. 2004, 126, 2288-2289. doi:10.1021/ja0395285

10. Crespo-Biel, O.; Lim, C. W.; Ravoo, B. J.; Reinhoudt, D. N.; Huskens, J. J. Am. Chem. Soc. 2006, 128, 17024-17032. doi:10.1021/ja0637705

11. Badjić, J. D.; Nelson, A.; Cantrill, S. J.; Turnbull, W. B.; Stoddart, J. F. Acc. Chem. Res. 2005, 38, 723-732. doi:10.1021/ar040223k

12. Lee, Y. C.; Lee, R. T. Acc. Chem. Res. 1995, 28, 321-327. doi:10.1021/ar00056a001

13. Lin, B.; Qing, X.; Liao, J.; Zhuo, K. Cells 2020, 9, 1022. doi:10.3390/cells9041022

14. Krachler, A. M.; Ham, H.; Orth, K. Proc. Natl. Acad. Sci. U. S. A. 2011, 108, 11614-11619. doi:10.1073/pnas.1102360108

15. Fasting, C.; Schalley, C. A.; Weber, M.; Seitz, O.; Hecht, S.; Koksch, B.; Dernedde, J.; Graf, C.; Knapp, E.-W.; Haag, R. Angew. Chem., Int. Ed. 2012, 51, 10472-10498. doi:10.1002/anie.201201114

16. Liang, J.; Li, K.; Liu, B. Chem. Sci. 2013, 4, 1377-1394. doi:10.1039/c2sc21792a

17. Feng, X.; Liu, L.; Wang, S.; Zhu, D. Chem. Soc. Rev. 2010, 39, 2411-2419. doi:10.1039/b909065g

18. Rochat, S.; Swager, T. M. ACS Appl. Mater. Interfaces 2013, 5 , 4488-4502. doi:10.1021/am400939w

19. Huskens, J.; Prins, L. J.; Haag, R.; Ravoo, B. J., Eds. Multivalency: Concepts, Research and Applications; John Wiley \& Sons: Hoboken, NJ, USA, 2018. doi:10.1002/9781119143505

20. Dreher, M. R.; Simnick, A. J.; Fischer, K.; Smith, R. J.; Patel, A.; Schmidt, M.; Chilkoti, A. J. Am. Chem. Soc. 2008, 130, 687-694. doi:10.1021/ja0764862

21. Kingery-Wood, J. E.; Williams, K. W.; Sigal, G. B.; Whitesides, G. M. J. Am. Chem. Soc. 1992, 114, 7303-7305. doi:10.1021/ja00044a057

22. Drożdż, W.; Walczak, A.; Bessin, Y.; Gervais, V.; Cao, X.-Y.; Lehn, J.-M.; Ulrich, S.; Stefankiewicz, A. R. Chem. - Eur. J. 2018, 24, 10802-10811. doi:10.1002/chem.201801552

23. Rodrigo, A. C.; Barnard, A.; Cooper, J.; Smith, D. K. Angew. Chem., Int. Ed. 2011, 50, 4675-4679. doi:10.1002/anie.201100019

24. Rajangam, K.; Behanna, H. A.; Hui, M. J.; Han, X.; Hulvat, J. F.; Lomasney, J. W.; Stupp, S. I. Nano Lett. 2006, 6, 2086-2090. doi:10.1021/nl0613555

25. Xu, Z.; Jia, S.; Wang, W.; Yuan, Z.; Ravoo, B. J.; Guo, D.-S. Nat. Chem. 2019, 11, 86-93. doi:10.1038/s41557-018-0164-y

26. Sandanaraj, B. S.; Vutukuri, D. R.; Simard, J. M.; Klaikherd, A. Hong, R.; Rotello, V. M.; Thayumanavan, S. J. Am. Chem. Soc. 2005, 127, 10693-10698. doi:10.1021/ja051947+

27. Bhaumik, S. K.; Patra, Y. S.; Banerjee, S. Chem. Commun. 2020, 56, 9541-9544. doi:10.1039/d0cc03644g

28. Knowles, J. R. Annu. Rev. Biochem. 1980, 49, 877-919. doi:10.1146/annurev.bi.49.070180.004305

29. Higgins, C. F.; Hiles, I. D.; Salmond, G. P. C.; Gill, D. R.; Downie, J. A.; Evans, I. J.; Holland, I. B.; Gray, L.; Buckel, S. D.; Bell, A. W.; Hermodson, M. A. Nature 1986, 323, 448-450. doi:10.1038/323448a0 
30. Yu, M.; Yao, Y.; Cui, B.; Sun, C.; Zhao, X.; Wang, Y.; Liu, G.; Cui, H.; Zeng, Z. ACS Appl. Nano Mater. 2019, 2, 48-57. doi:10.1021/acsanm.8b01583

31. Bhowmik, S.; Ghosh, B. N.; Marjomäki, V.; Rissanen, K. J. Am. Chem. Soc. 2014, 136, 5543-5546. doi:10.1021/ja4128949

32. Weitz, E. A.; Chang, J. Y.; Rosenfield, A. H.; Morrow, E. A.; Pierrre, V. C. Chem. Sci. 2013, 4, 4052-4060. doi:10.1039/c3sc51583d

33. Kurishita, Y.; Kohira, T.; Ojida, A.; Hamachi, I. J. Am. Chem. Soc. 2012, 134, 18779-18789. doi:10.1021/ja308754g

34. Ngo, H. T.; Liu, X.; Jolliffe, K. A. Chem. Soc. Rev. 2012, 41, 4928-4965. doi:10.1039/c2cs35087d

35. Hargrove, A. E.; Nieto, S.; Zhang, T.; Sessler, J. L.; Anslyn, E. V. Chem. Rev. 2011, 111, 6603-6782. doi:10.1021/cr100242s

36. Zuo, X.; Song, S.; Zhang, J.; Pan, D.; Wang, L.; Fan, C. J. Am. Chem. Soc. 2007, 129, 1042-1043. doi:10.1021/ja067024b

37. Tang, Z.; Mallikaratchy, P.; Yang, R.; Kim, Y.; Zhu, Z.; Wang, H.; Tan, W. J. Am. Chem. Soc. 2008, 130, 11268-11269. doi:10.1021/ja804119s

38. Liu, Z.; Chen, S.; Liu, B.; Wu, J.; Zhou, Y.; He, L.; Ding, J.; Liu, J. Anal. Chem. (Washington, DC, U. S.) 2014, 86, 12229-12235. doi:10.1021/ac503358m

39. Zhou, Q.; Swager, T. M. J. Am. Chem. Soc. 1995, 117, 12593-12602. doi:10.1021/ja00155a023

40. Parthasarathy, A.; Pappas, H. C.; Hill, E. H.; Huang, Y.; Whitten, D. G.; Schanze, K. S. ACS Appl. Mater. Interfaces 2015, 7, 28027-28034. doi:10.1021/acsami.5b02771

41. Cui, Q.; Yang, Y.; Yao, C.; Liu, R.; Li, L. ACS Appl. Mater. Interfaces 2016, 8, 35578-35586. doi:10.1021/acsami.6b12525

42. Zhao, Q.; Zhang, Z.; Tang, Y. Chem. Commun. 2017, 53, 9414-9417. doi:10.1039/c7cc04293k

43. Huang, H.; Tan, Y.; Shi, J.; Liang, G.; Zhu, J.-J. Nanoscale 2010, 2 , 606-612. doi:10.1039/b9nr00393b

44. Zhong, Y.; Yi, T. J. Mater. Chem. B 2019, 7, 2549-2556. doi:10.1039/c9tb00191c

45. Maity, D.; Li, M.; Ehlers, M.; Schmuck, C. Chem. Commun. 2017, 53, 208-211. doi:10.1039/c6cc08386b

46. Xu, Z.; Singh, N. J.; Lim, J.; Pan, J.; Kim, H. N.; Park, S.; Kim, K. S.; Yoon, J. J. Am. Chem. Soc. 2009, 131, 15528-15533. doi:10.1021/ja906855a

47. Li, X.; Guo, X.; Cao, L.; Xun, Z.; Wang, S.; Li, S.; Li, Y.; Yang, G. Angew. Chem., Int. Ed. 2014, 53, 7809-7813. doi:10.1002/anie.201403918

48. Nishizawa, S.; Kato, Y.; Teramae, N. J. Am. Chem. Soc. 1999, 121 , 9463-9464. doi:10.1021/ja991497j

49. Noguchi, T.; Shiraki, T.; Dawn, A.; Tsuchiya, Y.; Ngoc Lien, L. T.; Yamamoto, T.; Shinkai, S. Chem. Commun. 2012, 48, 8090-8092. doi:10.1039/c2cc33262k

50. Huang, L.-X.; Guo, Q.; Chen, Y.; Verwilst, P.; Son, S.; Wu, J.-B.; Cao, Q.-Y.; Kim, J. S. Chem. Commun. 2019, 55, 14135-14138. doi: $10.1039 / \mathrm{c} 9 \mathrm{cc} 08054 \mathrm{f}$

51. Xu, H.-R.; Li, K.; Jiao, S.-Y.; Pan, S.-L.; Zeng, J.-R.; Yu, X.-Q. Analyst 2015, 140, 4182-4188. doi:10.1039/c5an00484e

52. Ma, H.; Yang, M.; Zhang, C.; Ma, Y.; Qin, Y.; Lei, Z.; Chang, L.; Lei, L.; Wang, T.; Yang, Y. J. Mater. Chem. B 2017, 5, 8525-8531. doi:10.1039/c7tb02399e

53. Alcalde, E.; Mesquida, N.; Vilaseca, M.; Alvarez-rúa, C.; García-Granda, S. Supramol. Chem. 2007, 19, 501-509. doi:10.1080/10610270601132624

54. Ahmed, N.; Shirinfar, B.; Geronimo, I.; Kim, K. S. Org. Lett. 2011, 13, 5476-5479. doi:10.1021/ol202183t
55. Wang, D.; Zhang, X.; He, C.; Duan, C. Org. Biomol. Chem. 2010, 8 , 2923-2925. doi:10.1039/c004148c

56. Kwon, J. Y.; Singh, N. J.; Kim, H. N.; Kim, S. K.; Kim, K. S.; Yoon, J. J. Am. Chem. Soc. 2004, 126, 8892-8893. doi:10.1021/ja0492440

57. Maity, D.; Assaf, K. I.; Sicking, W.; Hirschhäuser, C.; Nau, W. M.; Schmuck, C. Chem. - Eur. J. 2019, 25, 13088-13093. doi:10.1002/chem.201901037

58. Winnik, F. M. Chem. Rev. 1993, 93, 587-614. doi:10.1021/cr00018a001

59. Ge, Y.; Wen, Y.; Liu, H.; Lu, T.; Yu, Y.; Zhang, X.; Li, B.; Zhang, S.-T.; Li, W.; Yang, B. J. Mater. Chem. C 2020, 8, 11830-11838. doi:10.1039/d0tc02562c

60. Biswas, R.; Naskar, S.; Ghosh, S.; Das, M.; Banerjee, S. Chem. - Eur. J. 2020, 26, 13595-13600. doi:10.1002/chem.202002648

61. Kumari, N.; Dey, N.; Jha, S.; Bhattacharya, S. ACS Appl. Mater. Interfaces 2013, 5, 2438-2445. doi:10.1021/am400063k

62. Černochová, J.; Branná, P.; Rouchal, M.; Kulhánek, P.; Kuřitka, I.; Vícha, R. Chem. - Eur. J. 2012, 18, 13633-13637. doi:10.1002/chem.201201444

63. Yang, C.; Mehmood, F.; Lam, T. L.; Chan, S. L.-F.; Wu, Y.; Yeung, C.-S.; Guan, X.; Li, K.; Chung, C. Y.-S.; Zhou, C.-Y.; Zou, T.; Che, C.-M. Chem. Sci. 2016, 7, 3123-3136. doi:10.1039/c5sc04458h

64. Haedler, A. T.; Misslitz, H.; Buehlmeyer, C.; Albuquerque, R. Q.; Köhler, A.; Schmidt, H.-W. ChemPhysChem 2013, 14, 1818-1829. doi:10.1002/cphc.201300242

65. Above $50 \mu \mathrm{M}$, the ratios were not calculated since the absorbance values were high.

66. Kalyanasundaram, K.; Thomas, J. K. J. Am. Chem. Soc. 1977, 99, 2039-2044. doi:10.1021/ja00449a004

67. Somerharju, P. Chem. Phys. Lipids 2002, 116, 57-74. doi:10.1016/s0009-3084(02)00020-8

68. Karpovich, D. S.; Blanchard, G. J. J. Phys. Chem. 1995, 99, 3951-3958. doi:10.1021/j100012a014

69. Lehrer, S. S. Subcell. Biochem. 1995, 24, 115-132. doi:10.1007/978-1-4899-1727-0_5

70. Birks, J. B. Photophysics of aromatic Molecules; Wiley-Interscience: New York, NY, USA, 1970; pp 1294-1295.

71. Chan, C. W.; Smith, D. K. Chem. Commun. 2016, 52, 37853788. doi:10.1039/c6cc00163g

72. Jiao, Q.; Liu, Q. Anal. Lett. 1998, 31, 1311-1323. doi:10.1080/00032719808002868

73. Gribble, F. M.; Loussouarn, G.; Tucker, S. J.; Zhao, C.; Nichols, C. G.; Ashcroft, F. M. J. Biol. Chem. 2000, 275, 30046-30049. doi:10.1074/jbc.m001010200 


\section{License and Terms}

This is an Open Access article under the terms of the Creative Commons Attribution License (https://creativecommons.org/licenses/by/4.0). Please note that the reuse, redistribution and reproduction in particular requires that the authors and source are credited.

The license is subject to the Beilstein Journal of Organic Chemistry terms and conditions:

(https://www.beilstein-journals.org/bjoc)

The definitive version of this article is the electronic one which can be found at:

https://doi.org/10.3762/bjoc.16.223 